\title{
Transferência de massa em sistemas de aeração por jatos bifásicos
}

\author{
Mass transfer in two-phase jet aeration systems
}

\author{
Iran Eduardo Lima Neto \\ PhD em Engenharia Hidráulica. Professor Adjunto do Departamento de Engenharia Hidráulica \\ e Ambiental da Universidade Federal do Ceará (UFC) - Fortaleza (CE), Brasil.
}

\section{Resumo}

A injeção de misturas de ar e água na forma de jatos bifásicos é bastante usada para a aeração artificial em tanques e corpos hídricos. No presente trabalho, foram realizados experimentos para investigar a transferência de massa induzida por estes jatos em um tanque de aeração. Os resultados forneceram eficiências de transferência de oxigênio padrão variando entre aproximadamente 5 e 9\%. Combinando-se os dados obtidos nesta pesquisa com os disponíveis na literatura, foram geradas correlações adimensionais para o coeficiente de transferência de massa em função da fração volumétrica de ar e do número de Froude densimétrico. Finalmente, os resultados foram aplicados a situações práticas envolvendo sistemas de aeração por jatos bifásicos.

Palavras-chave: aeração; bolhas; escoamento multifásico; jatos bifásicos.

\section{Abstract}

The injection of air-water mixtures through two-phase jets is widely used for artificial aeration in tanks and water bodies. In this study, experiments were conducted to investigate mass transfer induced by such jets in an aeration tank. The results provided standard oxygen transfer efficiencies ranging from about 5 to $9 \%$. Combining the results obtained here with those from previous investigations allowed to generate dimensionless correlations for the mass transfer coefficient as a function of the gas volume fraction and the densimetric Froude number. Finally, the results were applied to practical situations involving twophase jet aeration systems.

Keywords: aeration; bubbles; multiphase flow; two-phase jets.

\section{Introdução}

A injeção de misturas de gases e líquidos na água resulta em jatos borbulhantes ou jatos bifásicos do tipo gás-líquido que se espalham lateralmente à medida que ascendem em direção à superfície da água, conforme mostrado esquematicamente na Figura 1. Estes tipos de escoamentos ocorrem em muitas aplicações nas áreas de engenharia sanitária e ambiental (FAST \& LORENZEN, 1976; SUN \& FAETH, 1986; IGUCHI et al., 1997; MUELLER; BOYLE; PÖPEL, 2002; LIMA NETO et al., 2007; LIMA NETO, 2010, 2012).

Em sistemas de aeração por jatos bifásicos é importante determinar a taxa de transferência de massa das bolhas para a água, a qual é estimada usando a seguinte equação, derivada da lei de Fick (VON SPERLING, 1996; MUELLER; BOYLE; PÖPEL, 2002):

$$
\frac{d C}{d t}=K_{L} a\left(C_{s}-C\right)
$$

Sendo,

C a concentração de oxigênio dissolvido (OD) na água;

$\mathrm{C}_{\mathrm{s}}$ a concentração de saturação de oxigênio na água;

$\mathrm{K}_{\mathrm{L}}$ o coeficiente de transferência de massa;

a a área interfacial das bolhas dividida pelo volume do líquido em contato, ou, simplesmente, área interfacial específica.

Medições recentes do coeficiente de transferência de massa volumétrico $\left(\mathrm{K}_{\mathrm{L}} \mathrm{a}\right)$ têm sido fornecidas por Lima Neto, Zhu e Rajaratnam (2008d). Entretanto, tais experimentos foram limitados a misturas de ar e água injetadas horizontalmente em um tanque de aeração. Por outro lado, o estudo de Lima Neto, Zhu e Rajaratnam (2008b) foi conduzido em misturas de ar e água injetadas verticalmente em um tanque de aeração, foco do presente trabalho, mas não foram obtidas informações sobre $\mathrm{K}_{\mathrm{L}}$. Além disso, os experimentos foram realizados para números de Froude densimétricos relativamente baixos. Ressalta-se que este parâmetro tem sido recentemente apontado como o fator que controla 
a hidrodinâmica de jatos bifásicos do tipo gás-líquido (LIMA NETO, 2012). Outros estudos experimentais foram conduzidos por Sun e Faeth (1986), Kumar, Nikitopoulos e Michaelides, (1989), Iguchi et al. (1997) e Lima Neto (2010), mas estes tiveram foco no escoamento gerado por jatos bifásicos do tipo gás-líquido, sem obter valores para $\mathrm{K}_{\mathrm{L}} \mathrm{a}$. Por outro lado, os estudos de campo realizados por Fast \& Lorenzen (1976), Mueller, Boyle e Pöpel (2002) e Lima Neto et al. (2007) obtiveram valores para a eficiência de transferência de oxigênio (em percentagem do total injetado), mas também não forneceram valores para $\mathrm{K}_{\mathrm{L}} \mathrm{a}$. Ademais, os supracitados não obtiveram correlações para se estimar a taxa de transferência de massa em diferentes sistemas de aeração por jatos bifásicos.

O presente trabalho foi realizado usando o mesmo esquema experimental de Lima Neto, Zhu e Rajaratnam (2008b). Porém, os testes foram conduzidos com números de Froude densimétricos mais elevados com o intuito de investigar o seu impacto na transferência de massa induzida por jatos bifásicos em tanques de aeração. Dados adicionais de Lima Neto, Zhu e Rajaratnam (2008b, 2008d). também foram incluídos na análise para se obter correlações gerais que possam ser utilizadas em casos práticos.

\section{Material e métodos}

Os testes foram realizados em um tanque quadrado com base de $115 \mathrm{~cm}$ e altura de $80 \mathrm{~cm}$, conforme mostrado esquematicamente na Figura 1 (para mais detalhes, ver esquema experimental em LIMA NETO, 2010; LIMA NETO; ZHU; RAJARATNAM, 2008b). O tanque foi

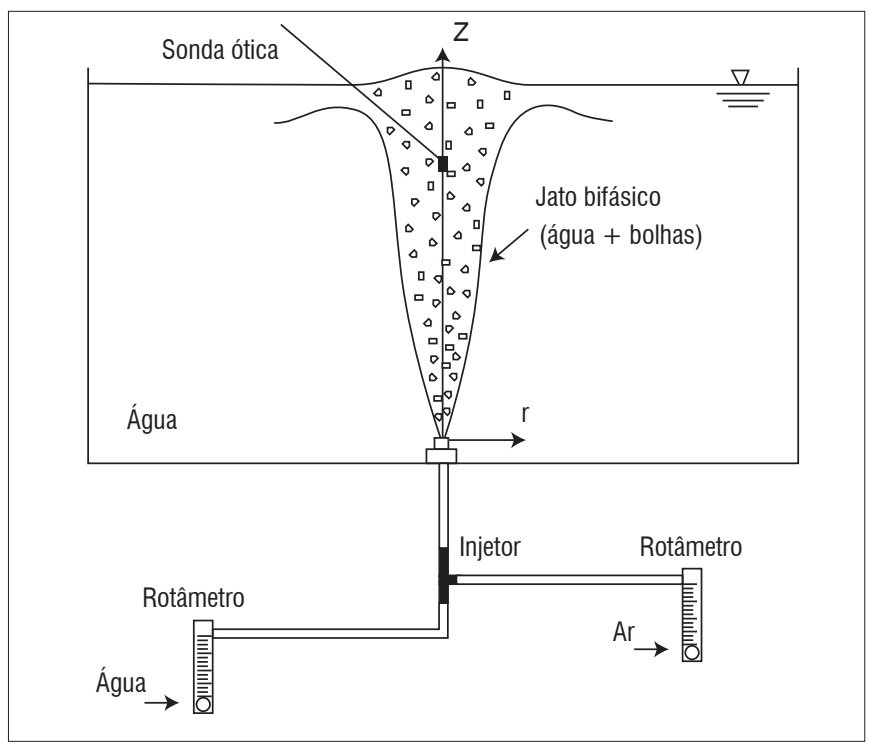

Figura 1 - Esquema de injeção de misturas do tipo gás-líquido na água, resultando em jatos borbulhantes ou jatos bifásicos (água + bolhas). preenchido com água até a altura de $76 \mathrm{~cm}$. O ar e a água foram bombeados respectivamente por meio de compressor e bomba centrífuga. As vazões volumétricas de ar $\left(Q_{\mathrm{g}}\right)$, e água $\left(Q_{\mathrm{l}}\right)$ foram ajustadas através de rotâmetros, sendo a mistura gás-líquido realizada no interior de um injetor, conectado a um tubo vertical de 25,4 mm, e descarregada por bocais circulares com diâmetros de saída (d) de 3 e 4 mm. Os bocais foram colocados no centro do tanque com suas saídas a uma altura de $45 \mathrm{~mm}$ acima da base do mesmo. A Tabela 1 apresenta um resumo das condições experimentais adotadas no presente trabalho, no qual $\varepsilon$ é a fração volumétrica de ar, Re é o número de Reynolds, e Fr é o número de Froude densimétrico, definidos pelas Equações 2 a 4, respectivamente:

$\varepsilon=Q_{\mathrm{g}} /\left(\mathrm{Q}_{\mathrm{g}}+\mathrm{Q}_{\mathrm{l}}\right)$

$\mathrm{Re}=\mathrm{Ud} / \mathrm{v}$

$\mathrm{Fr}=\mathrm{U} /\left(\mathrm{g}^{\prime} \mathrm{d}\right)^{1 / 2}$

sendo: U a velocidade do jato de água no bocal de descarga dada por $\mathrm{U}=\mathrm{Q}_{\mathrm{I}} /\left(\pi \mathrm{d}^{2} / 4\right)$, $v$ a viscosidade cinemática da água e g' a gravidade reduzida dada por g'=g( $\left.\rho_{1}-\rho_{a}\right) / \rho_{l}$, em que $\rho_{1}$ e $\rho_{a}$ são as densidades da água e do ar, respectivamente. Observa-se que todos os experimentos foram realizados com Re>8.000, condição crítica para produzir bolhas de tamanhos aproximadamente uniformes com diâmetros variando entre 1 e 4 mm, conforme sugerido por Lima Neto, Zhu e Rajaratnam (2008b, 2008d).

Um sistema de sonda ótica (RBI Instrumentation) foi utilizado para medir as propriedades das bolhas (Figura 1) — detalhes do referido sistema podem ser encontrados em Lima Neto, Zhu e Rajaratnam (2008a, 2008b, 2008c, 2008d). Os sinais captados pela sonda ótica foram processados para calcular a frequência $\left(\mathrm{f}_{\mathrm{b}}\right)$ e a velocidade $\left(\mathrm{u}_{\mathrm{b}}\right)$ das bolhas em distâncias radiais do centro do tanque de $\mathrm{r}=0,2$, 4 e $6 \mathrm{~cm}$ e a uma altura da saída do bocal $z=40 \mathrm{~cm}$. Nos experimentos com menores vazões de ar pode-se realizar uma comparação das frequências e velocidades das bolhas obtidas com a sonda ótica e a partir de imagens capturadas através de uma câmera de alta resolução do tipo CCD (Pulnix TM-1040), controlada por um sistema computacional (Streams 5, I. O. Industries Inc.). Em ambos os casos, as diferenças foram inferiores a 15\%, o que indica que as medições com a sonda ótica foram confiáveis. As imagens permitiram também confirmar o diâmetro das bolhas, os quais variaram entre aproximadamente 1 e $4 \mathrm{~mm}$. Assim, a seguinte equação (CHANSON, 2002; LIMA NETO; ZHU; RAJARATNAM, 2008A, 2008D) foi utilizada para estimar a área interfacial média (a):

$\mathrm{a}=4 \mathrm{f}_{\mathrm{b}} / \mathrm{u}_{\mathrm{b}}$

Tabela 1 - Condições experimentais adotadas para os jatos bifásicos.

\begin{tabular}{|c|c|c|c|c|c|c|}
\hline Testes & $\mathrm{d}(\mathrm{mm})$ & $Q_{g}\left(\mathrm{~cm}^{3} \cdot \mathrm{s}^{-1}\right)$ & $Q_{1}\left(\mathrm{~cm}^{3} \cdot \mathrm{s}^{-1}\right)$ & $\varepsilon$ & $\mathrm{Re}$ & $\mathrm{Fr}$ \\
\hline Experimento 1 & 3,0 & 16,7 & 25,0 & 0,40 & 10.616 & 20,6 \\
\hline Experimento 2 & 3,0 & 50,0 & 50,0 & 0,50 & 21.231 & 41,3 \\
\hline Experimento 3 & 4,0 & 16,7 & 33,3 & 0,33 & 10.616 & 13,4 \\
\hline Experimento 4 & 4,0 & 75,0 & 41,7 & 0,64 & 13.270 & 16,8 \\
\hline
\end{tabular}


Análise dimensional foi conduzida para normalizar os resultados e torná-los aplicáveis a outros sistemas de aeração por jatos bifásicos. Assim, usando o Teorema de Buckingham dos $\pi$ 's e assumindo que as forças devidas à viscosidade, tensão superficial e compressibilidade são desprezíveis comparadas às de inércia e gravidade, o coeficiente de transferência de massa pode ser expresso na seguinte forma adimensional:

$\left(K_{L} a\right) *\left(K_{L} a\right) d / U=\phi(\varepsilon, F r)$

(6)

\section{Resultados e discussão}

A Figura 2 mostra a distribuição radial de frequência das bolhas, velocidade e área interfacial (calculada a partir da Equação 5). Pode-se observar que os três parâmetros decrescem com a distância radial r, conforme observado por Lima Neto, Zhu e Rajaratnam (2008a) em experimentos com injeção de ar na água. Cabe salientar que apenas no experimento 1 não foram detectadas bolhas em $\mathrm{r}=6 \mathrm{~cm}$.
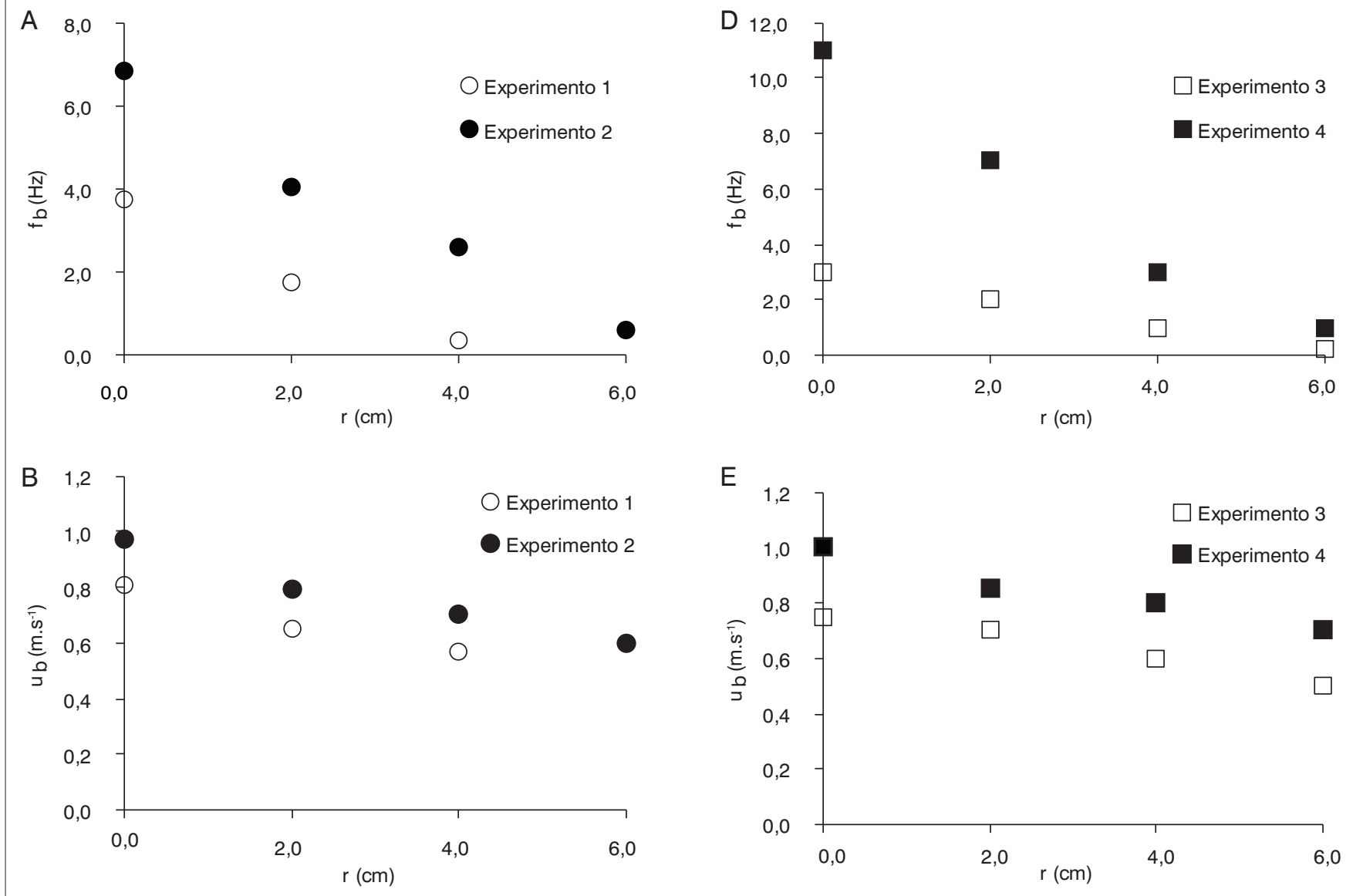

E
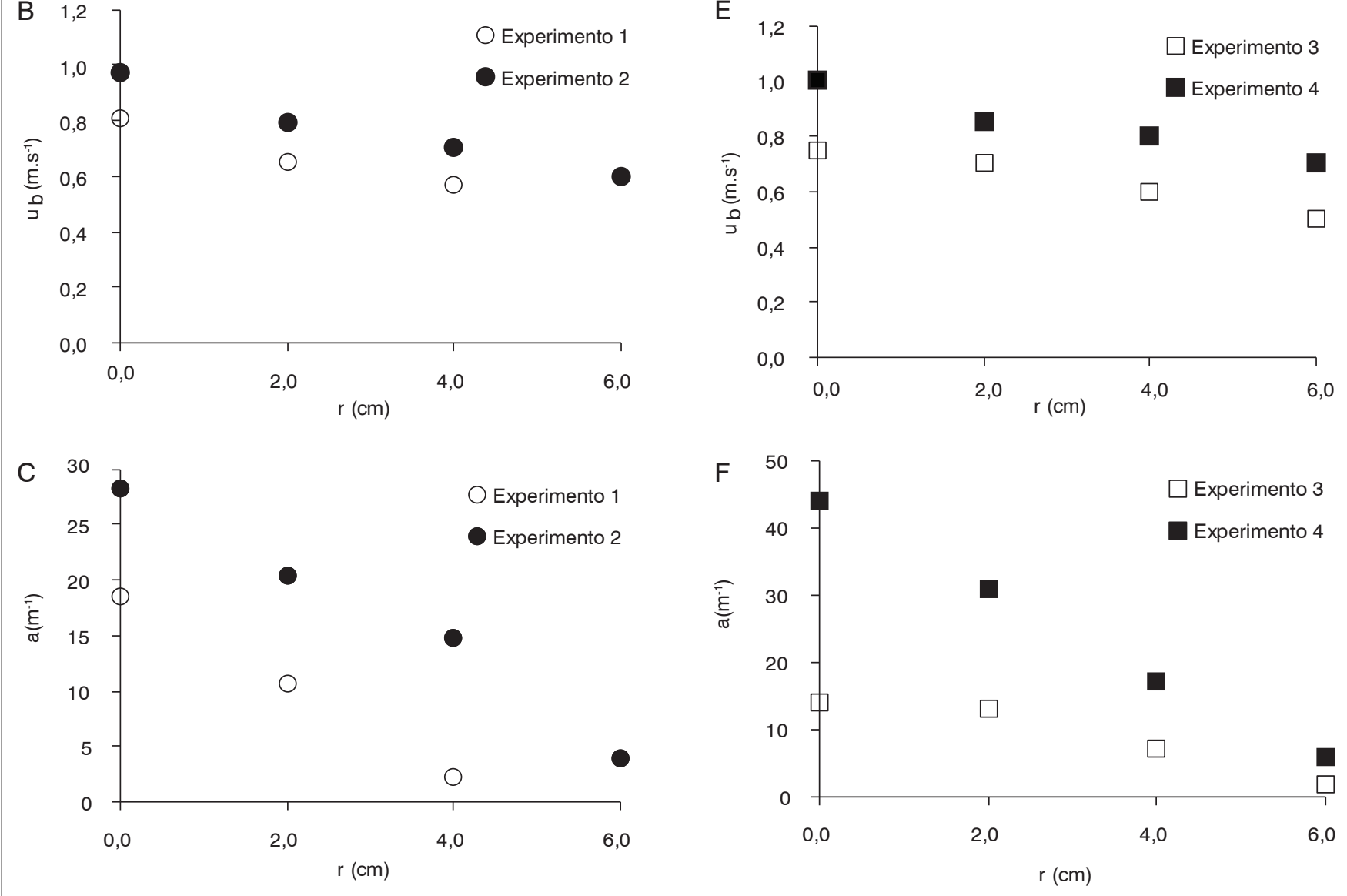

Figura 2 - Distribuição radial de frequência das bolhas, velocidade e área interfacial a uma altura da saída do bocal $z=40 \mathrm{~cm}$. 
Como as bolhas geradas no presente estudo tinham diâmetros aproximadamente uniformes entre 1 e $4 \mathrm{~mm}$, adotou-se um coeficiente de transferência de massa fixo $\mathrm{K}_{\mathrm{L}}=4 \times 10^{-4} \mathrm{~m} \cdot \mathrm{s}^{-1}$ (WÜEST; BROOKS; IMBODEN, 1992; LIMA NETO; ZHU; RAJARATNAM, 2008d). Utilizando este valor de $\mathrm{K}_{\mathrm{L}}$, os dados de área interfacial $a$ apresentados na Figura 2, e aplicando a Equação 1, considerando-se uma concentração inicial de $\mathrm{OD}(\mathrm{C})$ nula e uma concentração de saturação $\left(\mathrm{C}_{\mathrm{s}}\right)$ de $9 \mathrm{mg} \cdot \mathrm{L}^{-1}$ (a 20 C) (MUELLER; BOYLE; PÖPEL, 2002), obtém-se eficiências de transferência de oxigênio padrão, conforme definido por Von Sperling (1996), variando entre aproximadamente 5 e 9\%. A Tabela 2 apresenta uma comparação dessas eficiências com as obtidas de pesquisas anteriores em jatos bifásicos do tipo gás-líquido, os quais foram submetidos a diferentes profundidades de imersão do bocal de descarga. Os estudos de Lima Neto, Zhu e Rajaratnam (2008b, 2008d) estimaram a eficiência de transferência da mesma forma que a presente pesquisa, isto é, a partir do cálculo de $\mathrm{K}_{\mathrm{L}}$ a. Por outro lado, os estudos de Mueller, Boyle e Pöpel (2002) e Lima Neto et al. (2007) estimaram a eficiência medindo as concentrações de oxigênio dissolvido ao longo dos testes. Verifica-se que os valores obtidos neste trabalho são semelhantes aos calculados a partir dos dados de Lima Neto, Zhu e Rajaratnam (2008b), correspondentes a números de Froude densimétricos (Fr) variando entre 1 e 13, isto é, inferiores aos valores de Fr apresentados na Tabela 1, o que indica que este parâmetro por si só não afeta significativamente a eficiência de transferência de oxigênio para a água, assim como afetou a hidrodinâmica dos jatos borbulhantes no estudo de Lima Neto (2012). Uma possível explicação para tal situação é o fato de que aumentos em Fr resultam em velocidades da água mais elevadas, o que provavelmente provoca incrementos na frequência das bolhas proporcionais aos acréscimos na velocidade das mesmas. Assim, espera-se que a área interfacial a não seja significativamente alterada com elevados valores de Fr (Equação 5). Observa-se também que as eficiências de transferência de oxigênio tendem a aumentar com a profundidade de imersão do bocal, conforme relatado por Mueller, Boyle e Pöpel (2002). Entretanto, vale salientar que o estudo de Lima Neto et al. (2007), o qual utilizou oxigênio puro ao invés de ar difuso, apresentou eficiências de transferência bastante superiores às induzidas por injeção de ar na água (como nos testes supracitados), mesmo com profundidade de imersão relativamente baixa.

Conforme relatado anteriormente, uma análise dimensional foi realizada para normalizar os resultados e torná-los aplicáveis a outros sistemas de aeração por jatos bifásicos. A Figura 3 mostra o ajuste da
Equação 6 aos dados experimentais para a área interfacial a medida em r=0 (Figuras 2C e F), o que resultou na seguinte relação adimensional para o coeficiente de transferência de massa volumétrico na porção central dos jatos bifásicos:

$\left(K_{L} a\right)^{*}=1,7 \times 10^{-3} \phi^{1,53}$

sendo $\phi$ um parâmetro adimensional dado por $\pi=\varepsilon^{1 / 2} /$ Fr. A Figura 3 indica que a Equação 7 ajustou-se bem aos dados experimentais, com coeficiente de determinação $\mathrm{R}^{2}=0,944$. Observa-se que dados adicionais de Lima Neto, Zhu e Rajaratnam (2008b) também foram processados e incluídos na Figura 3. Isto mostra que a transferência de massa adimensional induzida pelos jatos bifásicos aumenta com $\varepsilon$ e com o inverso de Fr. É importante observar também que os coeficientes de transferência de massa volumétricos induzidos pelos jatos bifásicos horizontais de Lima Neto, Zhu e Rajaratnam (2008d) foram cerca de 30\% superiores aos obtidos no presente trabalho e no estudo de Lima Neto, Zhu e Rajaratnam (2008b), sugerindo que a injeção horizontal de misturas de ar e água seja vantajosa com relação à injeção vertical, uma vez que no primeiro caso as bolhas penetram inicialmente uma distância horizontal para depois ascenderem, o que resulta em menores velocidades e, consequentemente, maiores valores para a área interfacial $a$. Cabe ressaltar que na injeção horizontal ocorre separação parcial ou total do jato líquido, que tende a se deslocar na direção horizontal devido às forças de inércia e das bolhas, que tendem a ascender

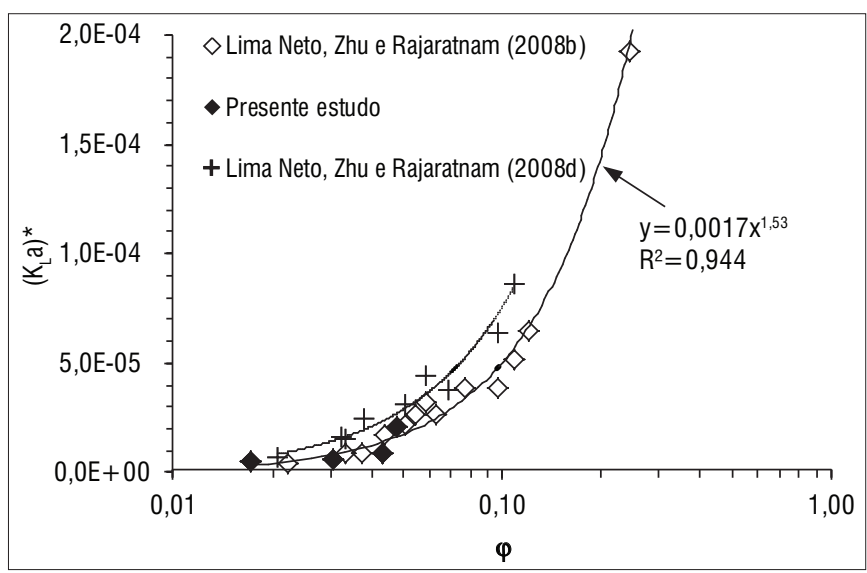

Figura 3 - Correlação adimensional para o coeficiente de transferência de massa volumétrico na porção central dos jatos bifásicos.

Tabela 2 - Eficiências de transferência de oxigênio obtidas neste estudo e em pesquisas anteriores para diferentes profundidades de imersão do bocal de descarga das misturas do tipo gás-líquido.

\begin{tabular}{lcc} 
Referência & Profundidade de imersão do bocal (m) & Eficiência de transferência de oxigênio padrão (\%) \\
\hline Lima Neto, Zhu e Rajaratnam (2008d) ${ }^{1,3}$ & 0,32 & $2-6$ \\
\hline Lima Neto, Zhu e Rajaratnam (2008b) ${ }^{1,3}$ & 0,76 & $4-8$ \\
\hline Presente estudo ${ }^{1,3}$ & 0,76 & $5-9$ \\
\hline Mueller, Boyle e Pöpel (2002) & $3,00-6,10$ \\
Lima Neto et al. $(2007)^{2,4}$ & 1,00 & $8-33$ \\
\hline
\end{tabular}

${ }^{1}$ Experimentos conduzidos utilizando ar comprimido. ${ }^{2}$ Experimentos conduzidos utilizando oxigênio puro. ${ }^{3}$ Eficiências de transferência estimadas a partir do cálculo de $K$ a. ${ }^{4}$ Eficiências de transferência estimadas a partir de medições das concentrações de oxigênio dissolvido. 


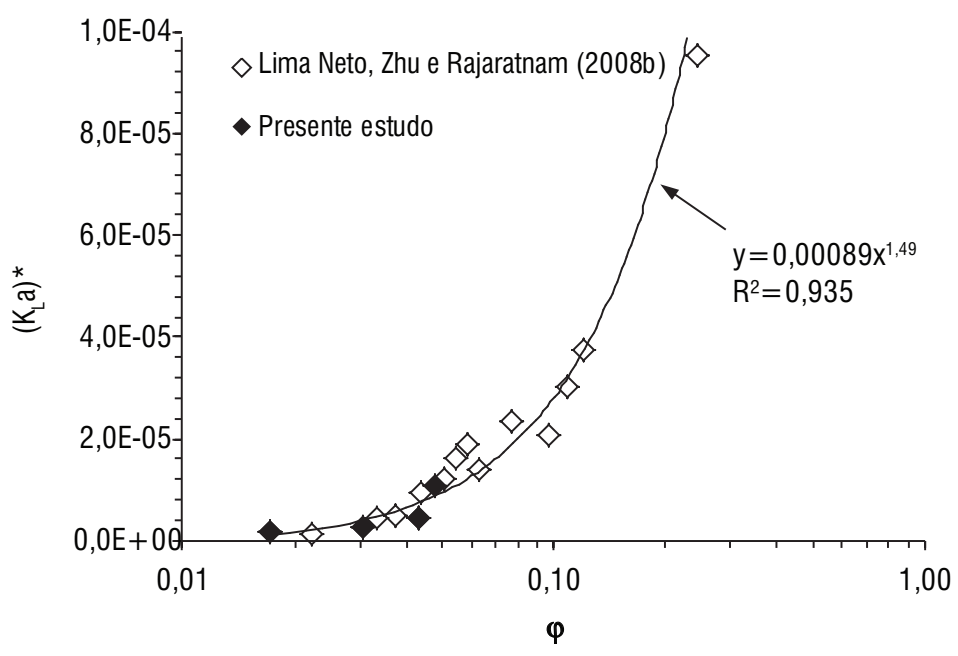

Figura 4 - Correlação adimensional para o coeficiente de transferência de massa volumétrico médio.

por conta das forças de gravidade, conforme descrito por Lima Neto, Zhu e Rajaratnam (2008d).

A Figura 4 mostra o ajuste da Equação 6 aos valores médios para a área interfacial $a$, obtidos para cada condição experimental (Figuras 2C e F), o que resultou na seguinte relação adimensional para o coeficiente de transferência de massa volumétrico:

$\left(\mathrm{K}_{\mathrm{L}} \mathrm{a}\right)^{*}=8,9 \times 10^{-4} \phi^{1,49}$

Sendo $\pi$ o mesmo parâmetro adimensional adotado na Equação (7). Na Figura 4 verifica-se que a Equação 8 ajustou-se bem aos dados experimentais, com coeficiente de determinação $\mathrm{R}^{2}=0,935$. Dados de Lima Neto, Zhu e Rajaratnam (2008b) também foram processados e incluídos, o que confirma novamente que a transferência de massa adimensional induzida pelos jatos bifásicos aumenta com e e com o inverso de Fr. Salienta-se que dados referentes aos experimentos de injeção horizontal de misturas de ar e água (LIMA NETO; ZHU; RAJARATNAM, 2008d) não constam na Figura 4, uma vez que foram coletados apenas na porção central dos jatos bifásicos.

Finalmente, é importante mencionar que as correlações dadas pelas Equações 7 e 8 foram obtidas para Re $>8.000 ; 2,2<$ Fr $<41,3$ e $0,05<\varepsilon<0,71$. Portanto, a aplicação delas em casos práticos deve ser restrita às faixas supracitadas.

\section{Aplicações}

Os resultados obtidos neste estudo podem ser usados para estimar a taxa de transferência de massa induzida por jatos bifásicos em tanques e corpos hídricos. Por exemplo: considerando um sistema de aeração por jatos bifásicos com bocais de $2,5 \mathrm{~cm}$ de diâmetro distribuídos uniformemente no fundo de um tanque, bem como vazões

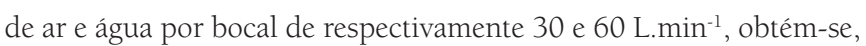

pela Equação 8, um valor para o coeficiente de transferência de massa volumétrico $\mathrm{K}_{\mathrm{L}} \mathrm{a}=14 \mathrm{~h}^{-1}$. Assim, pode-se utilizar a Equação 1 para estimar a taxa de transferência de oxigênio para a água. Cabe salientar que, neste caso simplificado, considerou-se a hipótese de mistura completa. No entanto, para cálculos mais detalhados, incluindo o efeito da hidrodinâmica dos jatos bifásicos, pode-se utilizar o modelo desenvolvido por Lima Neto (2012) e posteriormente aplicar a Equação 8. Por fim, é importante mencionar que a taxa de transferência aqui calculada deve ainda ser transformada para as condições reais de operação, incorporando o efeito dos diversos fatores intervenientes, como temperatura, concentração de oxigênio dissolvido e características do esgoto e do reator, conforme detalhado por Von Sperling (1996) e Mueller, Boyle e Pöpel (2002).

\section{Conclusões}

Nesta pesquisa, desenvolveu-se um estudo experimental com o intuito de investigar a transferência de massa gás-líquido em sistemas de aeração por jatos bifásicos. A partir dos resultados de área interfacial específica, foram obtidas eficiências de transferência de oxigênio padrão variando entre 5 e $9 \%$, as quais se compararam com dados disponíveis na literatura. Por meio de análise dimensional e compilação dos dados obtidos neste trabalho e em estudos anteriores, foi possível gerar correlações adimensionais para o coeficiente de transferência de massa em função da fração volumétrica de ar e do número de Froude densimétrico. Finalmente, apresentou-se uma aplicação prática das correlações obtidas em sistemas de aeração por jatos bifásicos.

\section{Agradecimentos}

O autor agradece aos professores Dr. David Zhu e Dr. Nallamuthu Rajaratnam pelo apoio na realização da pesquisa de pós-doutorado na Universidade de Alberta, que resultou no presente trabalho. 


\section{Referências}

CHANSON, H. (2002) Air-water flow measurements with intrusive phase-detection probes: can we improve their interpretation? Journal of Hydraulic Engineering, v. 128, n. 3, p. 252-255.

FAST, A.W. \& LORENZEN, M.W. (1976) Synoptic survey of hypolimnetic aeration. Journal of Environmental Engineering Division, v. 102, n. 6, 1 p. 1161-1173.

IGUCHI, M.; OKITA, K.; NAKATANI, T.; KASAI, N. (1997) Structure of turbulent round bubbling jet generated by premixed gas and liquid injection. International Journal of Multiphase Flow, v. 23, n. 2, p. 249-262.

KUMAR, S.; NIKITOPOULOS, D.N.; MICHAELIDES, E.E. (1989) Effect of bubbles on the turbulence near the exit of a liquid jet. Experiments in Fluids, v. 7, n. 7 , p. 487-494

LIMA NETO, I.E. (2012) Modeling the liquid volume flux in bubbly jets using a simple integral approach. Journal of Hydralic Engineering, v. 138, n. 2, p. 210-215.

LIMA NETO, I.E. (2010) Turbulência induzida por jatos bifásicos do tipo gás-líquido em tanques de aeração. Engenharia Sanitária e Ambiental, v. 15, n. 1, p. $75-82$

LIMA NETO, I.E.; ZHU, D.Z.; RAJARATNAM, N. (2008a) Air injection in water with different nozzles. Journal of Environmental Engineering, v. 134, n. 4, p. 283-294

LIMANETO, I.E.; ZHU, D.Z.; RAJARATNAM, N. (2008b) Bubbly jets in stagnant water. International Journal of Multiphase Flow, v. 34, n. 12, p. 1130-1141.
LIMA NETO, I.E.; ZHU, D.; RAJARATNAM, N. (2008c) Effect of tank size and geometry on the flow induced by circular bubble plumes and water jets. Journal of Hydraulic Engineering, v. 134, n. 6 , p. 833-842.

LIMA NETO, I.E.; ZHU, D.Z.; RAJARATNAM, N. (2008d) Horizontal injection of gas-liquid mixtures in a water tank. Journal of Hydraulic Engineering, v. 134, n. 12, p. 1722-1731.

LIMA NETO, I.E.; ZHU, D.Z.; RAJARATNAM, N.; YU, T.; SPAFFORD, M.; MCEACHERN, P. (2007) Dissolved oxygen downstream of an effluent outfall in an ice-covered river: Nnatural and artificial aeration. Journal of Environmental Engineering. v. 133, n. 11, p. 1051-1060.

MUELLER, J.A.; BOYLE, W.C.; PÖPEL, H.J. (2002) Aeration: principles and practice. New York: CRC Press.

SUN, T.Y. \& FAETH, G.M. (1986) Structure of turbulent bubbly jets - I. Methods and centerline properties. International Journal of Multiphase Flow, v. 12, n. 1, p. 99-114.

VON SPERLING, M. (1996) Princípios básicos do tratamento de esgotos. Princípios do tratamento biológico de águas residuárias, v. 2. Belo Horizonte: DESA-UFMG, ABES.

WÜEST, A.; BROOKS, N.H.; IMBODEN, D.M. (1992) Bubble plume modelling for lake restoration. Water Resources Research, v. 28, n. 12 , p. 3235-3250. 